\title{
LHomme
}

L'HOMME Revue française d'anthropologie

206 | 2013

Varia

\section{Système de mariages et terminologie de parenté chez les Mossi (Burkina Faso)}

Contribution à l'approche de la terminologie omaha

Marriage Systems and Kinship Terminology among the Mossi. A Contribution to the Approach Using Omaha Terminology

\section{Pierre-Joseph Laurent}

\section{OpenEdition}

\section{Journals}

Édition électronique

URL : http://journals.openedition.org/lhomme/24517

DOI : $10.4000 /$ lhomme. 24517

ISSN : $1953-8103$

\section{Éditeur}

Éditions de l'EHESS

\section{Édition imprimée}

Date de publication : 4 juin 2013

Pagination : $59-87$

ISSN : 0439-4216

Référence électronique

Pierre-Joseph Laurent, « Système de mariages et terminologie de parenté chez les Mossi (Burkina Faso) », L'Homme [En ligne], 206 | 2013, mis en ligne le 03 juin 2015, consulté le 02 mai 2019. URL : http://journals.openedition.org//homme/24517 ; DOI : 10.4000/lhomme.24517 


\title{
Système de mariages et terminologie de parenté chez les Mossi (Burkina Faso)
}

Contribution à l'approche de la terminologie omaha

\author{
Pierre-Joseph Laurent
}

Q

UICONQUE A PARTAGÉ l'existence de villageois mossi comprend la perplexité de l'ethnologue face à la complexité des relations de parenté qu'il découvre. On reste, en effet, toujours en deçà de la vérité lorsqu'on évoque le temps, la patience et l'imagination nécessaires pour appréhender les principes de la terminologie qui caractérisent leur système de parenté. Globalement, à partir d'un individu de référence, cette terminologie est effective sur quatre générations et sur au moins trois lignages, ce qui implique une mémoire s'étendant sur près d'un siècle et la nécessité de retenir l'exacte manière de s'adresser à des centaines de personnes.

Pour une meilleure approche, notre raisonnement partira de l'hypothèse selon laquelle les relations entre parents consanguins et alliés se fondent sur celle entre le neveu maternel et son oncle : un neveu maternel représente, pour son oncle, le remplacement de sa mère, puis, à la mort de celle-ci, il demande à cet oncle de la remplacer en lui cédant une de ses filles, qu'il appellera "maman»! Cette formule à première vue énigmatique circonscrit toutefois bien le problème, car elle fournit des pistes d'interprétation tout en maintenant à distance, dans un premier temps, d'autres caractéristiques de la terminologie qui risqueraient de nous égarer. Nous verrons qu'elle permet notamment de montrer comment les relations d'autorité, hiérarchiques et de compétition propres au patrilignage sont tempérées par les relations avunculaires reposant sur la collaboration et la confiance. Ou comment, en définitive, les conduites sociales induites par l'univers avunculaire, légitimées par la terminologie mossi, se superposent, en la tempérant, à l'idéologie du patrilignage.

Je remercie les membres du séminaire du Laboratoire d'anthropologie prospective de m'avoir donné l'opportunité, lors de la séance du 7 octobre 2011, de débattre des principaux arguments de ce texte. 


\section{État de la question}

Il s'agit donc de tenter de comprendre pourquoi, pour Ego (masculin dans le raisonnement), la fille du frère de la mère - la cousine croisée matrilatérale - est une mère pour lui, alors que le fils de la sœur du père - le cousin croisé patrilatéral - est un neveu, sachant que la terminologie est classificatoire, qu'elle regroupe sous le même vocable un ensemble de personnes (par exemple, la mère, la fille de l'oncle maternel et la fille de la fille de l'oncle maternel sont des mamans pour Ego). Ce mécanisme, maintes fois décrit, veut qu'autour d'Ego les cousins croisés basculent d'une ou de plusieurs générations; Floyd G. Lounsbury (1964) lui a donné le nom de skewing principle, c'est-à-dire de fusion oblique.

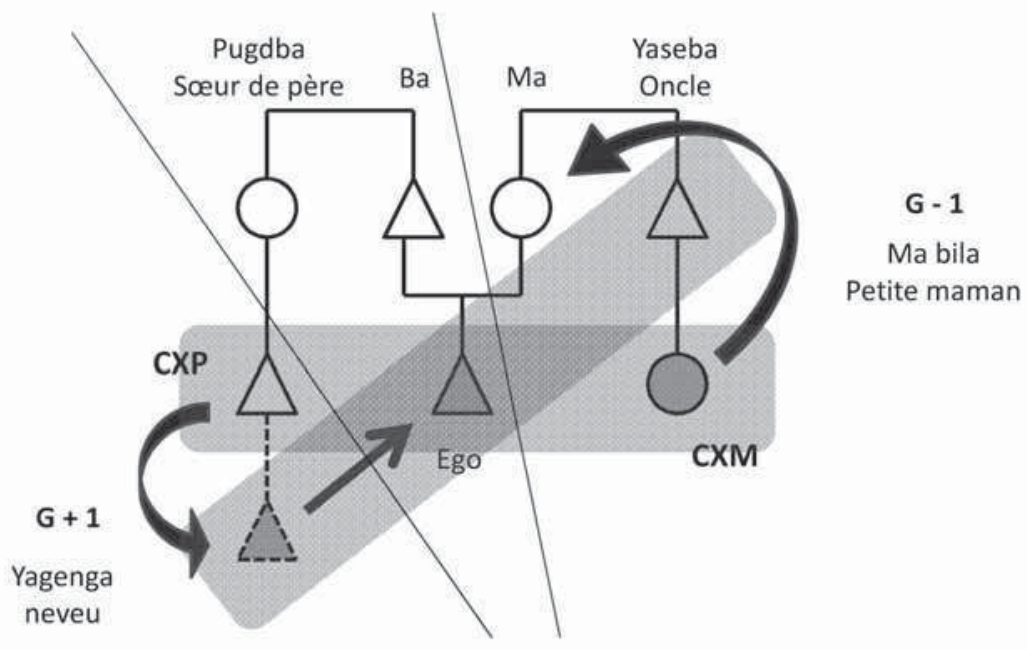

Lignage $2 \quad$ Lignage $1 \quad$ Lignage 3

I - Terminologie omaha : la fusion oblique (skewing principle)

La terminologie omaha et, plus largement, les systèmes crow et omaha sont connus des anthropologues depuis la fin du XIX siècle, surtout à partir des travaux que leur ont consacrés Alfred L. Kroeber (1909), Robert H. Lowie (1928: 267) Alfred R. Radcliffe-Brown (1952). Floyd G. Lounsbury, dans son analyse formelle des terminologies de parenté crow et omaha (1964), exposait trois grands traits pour les caractériser, dont la règle de projection oblique. Dans un style incisif, Rodney Needham, qui alla jusqu'à remettre en question la catégorie même de terminologie omaha ${ }^{1}$, précisait, à propos de l'article de Lounsbury, que ces analyses :

1. «Ainsi, les Iatmul, les Miwok, les Nyoro, les Thado, pour ne citer qu'eux, ont tous été assimilés sur la base de leurs terminologies Omaha, alors qu'un simple coup d'œil suffit à montrer que leurs formes de classification sociale [...] sont systématiquement différentes »(Needham 1977 : 114-115). 
"quoiqu'elles se donnent toutes les apparences de la rigueur algébrique et l'exactitude scientifique, elles n'en demeurent pas moins très simples quant au fond [...]. [L'] analyse compliquée des terminologies de type Crow - Omaha de Lounsbury n'apporte rien à celle de Tax, qui lui est même supérieure" (Needham 1977: 29). Françoise Héritier (1981)² a traité de cette question pour la société samo, voisine des Mossi; elle expliquait que : "Naturellement, la compréhension de l'armature logique du système et des équivalences telles qu'elles sont restituées par l'application de ces trois règles [énoncées par Lounsbury] ne dit rien de l'impérieuse nécessité qui fait que le système existe». Et de poursuivre : "Il reste cependant que si l'on comprend bien comment un individu fait l'apprentissage de ce système et l'utilise dans sa vie quotidienne, la genèse même et la raison d'être du système demeurent insaisissables" (Héritier 1996 : 63-64). Elle avançait également, pour rendre compte de la fusion oblique, que le système omaha serait l'expression la plus accomplie de la domination des femmes par les hommes (Ibid. : 66-67); une explication qui, nous le verrons, ne nous semble pas être la meilleure porte d'entrée pour rendre compte de la terminologie de parenté mossi. Concernant la fusion oblique, Maurice Godelier indiquait, quant à lui : «malgré les efforts de nombreux théoriciens de la parenté recourant à différentes approches formelles, linguistiques ou mathématiques, personne n'a encore fourni d'explication satisfaisante de la raison d'être de ce principe...» (2004 : 217).

Fort de ces mises en garde, nous nous garderons de proposer à notre tour notre solution au problème. Nous montrerons plutôt que les pistes ouvertes par Michael Houseman et Carlo Severi, à propos des Iatmul, nous semblent fécondes si on les compare au cas mossi (soulignant, par cette comparaison, qu'il existe bien des correspondances d'ordre structurel), notamment lorsqu'ils expliquent :

«Le système de mariage iatmul [...] ne réside donc ni en l'hypothèse d'une structure sociale idéale, ni en l'occurrence fréquente de tel ou tel type de mariage - l'application méthodique d'une règle ou d'une recette -, mais en une articulation la plus systématique possible, variable selon les circonstances, de deux principes d'“alliance contraire" " (Houseman \& Severi 2009: 113) ${ }^{3}$.

\section{Cf. également Francis Zimmermann (1993: 116-129).}

3. À ce propos, il est instructif de situer cet ouvrage dans le prolongement de l'article «Structures réticulaires de la pratique matrimoniale» de Michael Houseman et Douglas R. White (1996), où les auteurs précisent que l'analyse d'un système d'alliance ne demande pas "une mise à l'écart des terminologies de parenté, des règles de prohibition ou de préférence, etc., mais plutôt leur révaluation en fonction d'une prise en compte systématique des comportements réels ». Et de préciser plus loin : "Autrement dit, ce n'est que lorsque les registres classificatoires, normatifs et comportementaux du fonctionnement matrimonial sont envisagés comme nécessairement mais indirectement reliés les uns aux autres qu'une interrogation à la fois plus approfondie et plus réaliste de leur interrelation devient possible" (Ibid.: 79). 
Suivant cette proposition, nous postulons que pour démêler la terminologie de parenté mossi, sans s'égarer face à tant de complexité, il faut l'aborder en se laissant guider par la société elle-même. Cela permet de comprendre que, pour les Mossi, la terminologie ne se rapporte pas strictement aux indications relatives au mariage, mais plutôt à la manière de mettre en scène les comportements rituels qui règlent avec minutie les relations des uns avec les autres. Car ce sont ces relations, aux conduites ainsi réglementées par la terminologie, qui orienteront les projets d'alliances matrimoniales. De sorte que, en plus de l'importance accordée aux théories des informateurs ${ }^{4}$, on se doit de prendre en considération le contexte ethnographique au sens large: la perception de la personne humaine, l'ensemble des types d'alliance en ouvre chez les Mossi, les rituels liés aux étapes de la vie, ainsi que la nature de chacune des relations sociales valorisées au sein de la famille et entre les lignages. Nous voudrions démontrer que ces dimensions d'ordre contextuel peuvent rendre effectivement compte du fonctionnement d'une forme de terminologie regroupée sous la vaste catégorie omaha. Mais, pour cela, posons d'emblée qu'il convient de porter un double regard sur la société, en décrivant d'abord la famille mossi de l'intérieur, sans toutefois s'y laisser enfermer, afin de pouvoir, dans le même élan, relater les relations entre les lignages, et entre ceux-ci et les pouvoirs politiques locaux. D'autres méthodes conduiraient rapidement à des débats stériles, des aberrations, des supputations et, le plus souvent, à des impasses, là où les villageois, à la différence des ethnologues (et des enfants mossi), s'y retrouvent pourtant aisément.

\section{Approche contextuelle des relations de parenté et d'alliance}

Les éléments de contexte à prendre en considération pour envisager d'appréhender la terminologie de parenté mossi sont, surtout, la personne humaine telle qu'elle est perçue, les types d'alliance dans leur ensemble, les rituels liés aux étapes de la vie, ainsi que les relations sociales valorisées au sein de la famille et entre les lignages.

\section{La construction de la personne humaine}

Le premier élément contextuel que nous considérerons est celui qui concerne la construction de la personne humaine et sa perception chez les Mossi. Ce sujet étant particulièrement vaste et complexe ${ }^{5}$, il ne s'agira

4. Dans le sens donné par Viveiros de Castro lorsqu'il suggère que «la pensée indigène doit être prise - si on veut la prendre sérieusement - comme une pratique de sens : comme dispositif autoréférentiel de production de concepts..." (2009: 168).

5. Sur ce sujet, cf. par exemple : Étienne Poulet (1970); Amadé Badini (1979); Doris Bonnet (1988). 
ici que d'attirer l'attention sur les éléments offrant quelques clés pour comprendre le fonctionnement de la terminologie mobilisée par les Mossi. Par exemple, la façon dont ils conçoivent la personne humaine permet de comprendre pourquoi la cousine croisée matrilatérale n'est pas seulement et simplement perçue «comme» une maman, mais est bien une maman. Or, la terminologie requise dans cet exemple - ma (la mère d'Ego) et ma bila (sa cousine croisée matrilatérale) - exprime le cœur de l'identité coutumière mossi, à savoir la permanence de l'être, selon laquelle il n'y a pas d'êtres neufs mais toujours les mêmes qui reviennent ${ }^{6}: m a$ et ma bila sont semblables, en ce sens qu'elles peuvent être le vecteur du retour du même ancêtre.

En effet, chez les Mossi, une composante de la personne, l'instance dénommée siiga ("force vitale»), représente généralement, à l'issue de plusieurs transformations (siiga, tuulé, kiima), un ancêtre (kiima, pl.: kiimse). Autrement dit, le siiga, véritable double de la personne humaine, correspond non seulement à l'essence même de la vie, mais aussi à la présence de nen-lebende, le regard retourné, le monde invisible. La référence aux ancêtres évoque la loi du groupe, maîtrisée par les aînés, celle des "pères morts". La naissance comme la mort équivalent à des rituels de passages, où globalement ce sont les mêmes êtres qui, changeant de statut, reviennent parmi les vivants ou partent vers la cour des ancêtres (kiimkulugo). La collectivité apparaît dès lors immuable, figée, établie pour une longue durée et, à la faveur d'une suprême ruse, le groupe des vivants se perpétue comme identique à lui-même : le rituel des funérailles symbolise l'accouchement ${ }^{7}$ et l'on dit d'une femme qui accouche "qu'elle a son tombeau ouvert». Tout se passe donc comme s'il n'y avait pas vraiment d'êtres neufs chez les Mossi, mais d'anciens êtres qui, à l'issue d'un séjour dans la cour des ancêtres, reviennent prendre leur place parmi les vivants.

6. Le baga (le «devin») est l'exemple parfait de cette "immuable permanence». Lors de son intronisation, la société célèbre ses funérailles, c'est-à-dire la mort (le départ) des instances de sa personne héritées de ses ascendants afin de libérer le chemin pour qu'il puisse devenir le réceptacle du tibo, c'est-à-dire l'esprit même de la divination. Il faut savoir que cet esprit, à la suite d'épreuves qu'il a fait subir aux humains, a choisi cette personne pour figurer parmi les vivants. Le baga incarne la permanence de l'être puisque, au-delà des générations, il est l'esprit intemporel du tibo. Dans la société mossi, le devin (baga) possède, entre autres fonctions, celle de tempérer les ardeurs sorcières. Situé en dehors de la mêlée des sentiments humains, réputé inattaquable par les sorciers car d'essence surnaturelle, le baga ne connaît ni désir, ni peur, ni jalousie. Dès lors, fort de sa capacité à gouverner l'invisible, perçu comme un ultime recours, le baga constitue un contrepouvoir face aux forces destructrices de la collectivité que sont censés figurer les envieux, les jaloux, les intrigants. Lorsque les autres possibilités ne suffisent plus à maintenir la paix, le baga, par la violence symbolique qu'il maîtrise - pouvant aller jusqu'à induire la mort -, s'impose alors à tous l'entente (wuum taaba), c'est-à-dire par-delà le conflit, l'idéologie du silence qui est aussi la paix fondée sur le consensus.

7. Le tombeau lui-même symbolise le corps de la femme qui accouche. 
Cela caractérise la «transsubstantiation partielle», car c'est plus exactement d'une parcelle d'ancêtre, le segre, qu'il est ici question (cf. Lallemand 1978), et conduit à concevoir la stabilité du segment de lignage (buudu) au-delà de la succession des générations et de la séparation inhérente à la mort. Ajoutons enfin que l'ancêtre "revient" chez un de ses descendants en ligne agnatique et qu'il peut revenir chez plusieurs personnes simultanément (Bonnet 1988: 88).

\section{Une société guerrière}

Les Mossi constituent historiquement une société guerrière où la violence tant institutionnelle ${ }^{8}$ que physique était quotidienne. Ce fait n'est peut-être pas sans rapport avec une terminologie de type omaha, dans la mesure où un trait de la terminologie consiste, chez les Mossi, à imposer une forme de paix (avunculaire) transversale aux unités de résidence, qualifiée de zemes taaba (" ajuster ensemble»).

Membres d'une société à État - hiérarchisée et centralisée -, expansionniste, les Mossi terrorisèrent, par la supériorité de leur art guerrier, les populations paysannes voisines organisées en sociétés lignagères (désignées par eux comme étant les autochtones). Il leur fut ainsi aisé de s'imposer et de constituer leur territoire: le Moogo. Lorsque les cavaliers mossi envahirent le plateau central, ils possédaient déjà Wende, divinité guerrière. Ce Dieu cavalier accorda aux descendants du prince fondateur Ouedraogo le principe de commandement, le naam: les Mossi d'origine noble (nakomsé) naissent avec le désir et le pouvoir de commander les autres, de devenir un jour le naaba (le "chef») d'une unité territoriale.

\section{Un système d'alliance semi-complexe : principes généraux}

Suivant, comme nous l'avons vu, la terminologie de parenté appelée " omaha ", le système d'alliance mossi est semi-complexe, c'est-à-dire qu'il repose sur un ensemble de règles qui déterminent les groupes de personnes interdites au mariage ${ }^{9}$ : le mariage est fondé sur un principe d'interdits consanguins. La société mossi est dès lors strictement exogamique, l'exogamie étant en principe recherchée sur trois générations entre les personnes ayant un lien de parenté, quels que soient les intermédiaires qui les relient; huit lignages sont donc généralement prohibés : ceux de $F, M$, FM, MM, FFM, FMM, MFM ; MMM ${ }^{10}$. La polygynie est autorisée.

8. Celle produite par les royautés mossi (cf. Michel Izard 1992).

9. Cf., par exemple : Françoise Héritier (1981) ; Virginie Vinel (2005) ; Francis Zimmerman (1993).

10. À côté de cette règle générale, il en existe d'autres (re)étudiées dernièrement par Virginie Vinel : «En outre, un homme ne peut prendre comme co-épouse la sœur de mêmes père et .... ... 
Dans l'Oubritenga, où j'ai mené l'essentiel de cette enquête, comme l'a montré Suzanne Lallemand, "l'absence d'intermariages des Mossi "purs" avec le groupe des Peuls et celui, casté, des forgerons, accentue encore la tendance à l'exogamie » (1977: 156-157). Toutefois, si la règle d'exogamie favorise l'extension permanente des réseaux d'alliance, elle n’implique pas nécessairement que l'aire des relations matrimoniales soit très étendue. Ainsi, en 1971, Jean-Marie Kohler montrait, quant à lui, que : «Les mille trois cents femmes mariées à Pilimpikou sont originaires de près de quatre-vingt-dix localités, situées en majorité à moins de $25 \mathrm{~km}$ de Pilimpikou " (1971 : 181); ces observations corroborées par d'autres, plus récentes, mettent en évidence une assez nette endogamie géographique ${ }^{11}$. Il convient néanmoins de préciser que, le plus souvent, l'homme trouve son ou ses épouses en dehors de son propre village. Ajoutons, enfin, que la descendance est patrilinéaire (à la naissance l'enfant appartient aux hommes, ici au lignage du mari; si la femme quitte la résidence de son mari, elle doit laisser ses enfants) et que la résidence est patrilocale (le couple marié s'installe dans le lieu de résidence de l'époux), avec une certaine propension avunculocale.

\section{Alliances principales et secondaires}

Avant de rendre compte de façon plus détaillée des trois types d'alliance rencontrés chez les Mossi, soulignons que les anthropologues différencient souvent les mariages principaux des mariages secondaires. Or, selon notre point de vue, c'est sans distinction l'ensemble des alliances qu'une personne a connues dans son existence qui doit être analysé : l'alliance principale et ses règles très officielles n'épuisent pas la question lorsqu'on sait que les autres alliances, dites secondaires, ne le sont pas particulièrement pour les personnes qui les contractent ou qui doivent s'y soumettre.

Chez les Mossi, les formes prises par les alliances coutumières permettent à tout adulte, quel que soit son âge, d'obtenir, tôt ou tard, un conjoint (plusieurs dans le cas d'hommes polygynes) et de rester marié toute sa vie. La plupart des femmes, si elles sont généralement contraintes à un premier mariage coutumier, officiel (souvent voulu par les aînés, voire par les aînées), connaissent pourtant une véritable carrière matrimoniale en se

[Suite de la note 10] mère que sa femme, mais il peut épouser une demi-sœur agnatique ou une sœur classificatoire de celle-ci. Un fils peut se marier à une agnate d'une femme de son père et deux frères réels peuvent s'unir à des femmes du même lignage [...]. Une femme, quant à elle, ne peut se marier, ni avoir de rapports sexuels avec un agnat de son époux [sauf lévirat]" (2000: 207).

11. Virginie Vinel explique que: «Dans ce quartier sikoomse, l'endogamie locale est patente. En effet, sur les 182 alliances recensées [...] 155 (85\%) [ont été contractées avec une personne résidant] dans un rayon de dix kilomètres " (2000 : 209). Cf. aussi Françoise Imbs (1987 : 96-103). 
remariant plusieurs fois, en fonction des aléas de la vie mais aussi de leurs propres choix, ruptures, fuites et enlèvements étant fréquents. Quant aux hommes, lorsqu'ils sont polygames, ils peuvent se retrouver mariés avec un groupe de femmes qui, pour les raisons que l'on vient d'évoquer, varie fortement avec le temps. De ce fait, chaque membre du groupe s'assure une place dans la société, même lorsqu'il ne sera plus productif et devra alors être pris en charge par la nouvelle génération. Autrement dit, pour la société coutumière mossi, il n'y a pas de place viable à long terme pour un célibataire : tout adulte se doit d'être marié, qu'il soit jeune ou vieux, beau ou laid, fort ou faible, fécond ou stérile... Cela constitue le seul statut acceptable et la seule possibilité, donnée à des moments particuliers de la vie de chacun, d'assurer sa survie ${ }^{12}$.

Du point de vue des personnes, ce sont les aînés qui recourent au type d'alliance dite principale (laquelle peut appartenir indistinctement aux trois groupes décrits dans le point suivant), selon des critères stratégiques, et en fonction des opportunités et possibilités qui se présentent, pour marier les jeunes qui doivent s'y soumettre dans l'intérêt des lignages. Par la suite, si ces personnes contractent d'autres alliances, secondaires, elles pourront le faire de leur propre volonté (sentiments amoureux et/ou de stratégies de survie, par exemple) et non plus uniquement en fonction des lignages.

Ce sont donc le contexte et la trajectoire matrimoniale de chacun qui qualifient les alliances de principales ou de secondaires, et non un classement d'ordre hiérarchique. Ce qui importe davantage est que ces alliances revêtent différentes formes, réparties en trois groupes, chacune répondant à un type de relation précis avec un lignage allié ou en voie de le devenir. Les chefs de lignage (buudukasma) et/ou les jeunes garçons sauront utiliser à sa juste valeur chaque forme d'alliance dans la quête d'une épouse. De leur côté, les femmes mariées renforceront leur autorité et leur pouvoir dans la cour de leurs maris par la conclusion d'alliances impliquant des jeunes filles de leurs propres lignages (filles de frères).

\section{Le système de mariage mossi : trois groupes d'alliances forment un tout cohérent}

Après avoir montré qu'il n'y a pas lieu d'établir de distinction entre alliances principales et secondaires, nous nous proposons d'envisager l'ensemble des formes d'alliances mossi en un système cohérent, à partir des quelque neuf exemples observés sur notre terrain, dans le village de 
Kulkinka. Mais, pour ne pas être réductrice, cette démarche doit parvenir à considérer ces alliances globalement et non séparément, selon une échelle de valeurs, par exemple ${ }^{13}$. On sait que des alliances très spécifiques telles que le pog siuuré ${ }^{14}$ ont parfois servi de référence pour penser l'ensemble du système mossi, tout comme certaines études ne se sont attachées qu'à des aspects particuliers inhérents à ces types alliance ${ }^{15}$. Or, à mon sens, ces approches ne font ressortir l'articulation globale de ce système que partiellement (Vinel 2000, 2005).

Selon notre hypothèse, une logique d'ensemble se dégage du système de mariage mossi, historiquement fondée sur la nécessité d'optimiser les chances d'obtenir des épouses pour les hommes du lignage, et reposant, en même temps, sur deux principes apparemment contradictoires : l'un, d'ouverture vers de nouveaux alliés et l'autre, de repli sur soi-même en reproduisant d'anciennes alliances. Cette manière d'envisager l'alliance mossi se rapproche une fois encore des analyses de Michael Houseman et Carlo Severi sur les Iatmuls : "Le système de mariage iatmul [...] ne réside ni en l'hypothèse d'une structure sociale idéale [...], mais en une articulation la plus systématique possible, variable selon les circonstances, de deux principes d'alliances "contraires" " $\left(2009\right.$ : 113) ${ }^{16}$.

Cette logique ambivalente permet ainsi d'identifier deux types d'alliance, différents mais complémentaires : les alliances du premier type (pog belongo, pog kuuni), qui portent essentiellement sur la recherche de nouveaux alliés, afin d'élargir le réseau des lignages donateurs avec l'objectif de trouver des épouses pour les fils de son propre lignage; les alliances du deuxième type (yir paga et ma bila), qui consistent essentiellement à remplacer des épouses âgées et donc à reproduire une parenté déjà initiée par un belongo (une alliance de premier type). Ces alliances du deuxième groupe sont généralement les plus nombreuses. Le belongo, pour sa part, ouvre de nouvelles perspectives lorsque les autres possibilités de mariages "au plus proche" (yir paga et ma bila) s'amenuisent (à la suite, par exemple, de différends, assez fréquents, avec les lignages alliés).

Tout se passe donc comme si, pour trouver des épouses aux fils d'un même segment de lignage, il fallait à la fois ouvrir de nouveaux chemins de parenté, en concluant des pactes inédits d'alliances avec les lignages

13. Dans son ouvrage, Jean-Marie Kohler (1971) reste plutôt discret sur ce point dans son chapitre consacré aux alliances chez les Mossi.

14. Une alliance plutôt valorisée au sein des lignages naam (noblesse moaga), cf. Marc-Éric Gruénais (1985).

15. Cf. la question des ré-enchaînements des alliances, où les alliés sont aussi des parents.

16. Voir notamment les précisions apportées par la note 19 de la même page. 
jusqu'alors inconnus mais qui deviendront ensuite des alliés, et mettre tout en ouvre pour reproduire, génération après génération, les accords d'alliance conclus préalablement avec les lignages traditionnellement donneurs d'épouses.

À côté de ces deux premiers types d'alliance étroitement associés, s'en trouve un troisième. Par la diversité des formes d'alliance qu'il regroupe (sukiri, pokokôré, pog siuuré, teke teke, baga tibo), il offre la possibilité à des hommes et des femmes de résoudre un problème d'alliance (difficulté à trouver une épouse pour un groupe particulier, stérilité, fuite d'une épouse, décès d'un conjoint...), sachant que chez les Mossi, comme nous l'avons vu, il n'y a pas de place pour les célibataires ${ }^{17}$. Il convient de garder en mémoire que seule l'alliance permet à chacun, à des moments particuliers de la vie, d'assurer sa survie. Son rôle consiste aussi à assurer la sécurité sociale et économique des catégories de personnes considérées, durablement ou provisoirement, comme les plus faibles pour un groupe (les plus vieux, notamment). Cette recherche de sécurité repose sur trois principes: la solidarité transgénérationnelle, les alliances secondaires (l'héritage par des cadets des épouses plus âgées d'un père ou d'un frère aîné décédé) et certains aspects des trajectoires matrimoniales ${ }^{18}$.

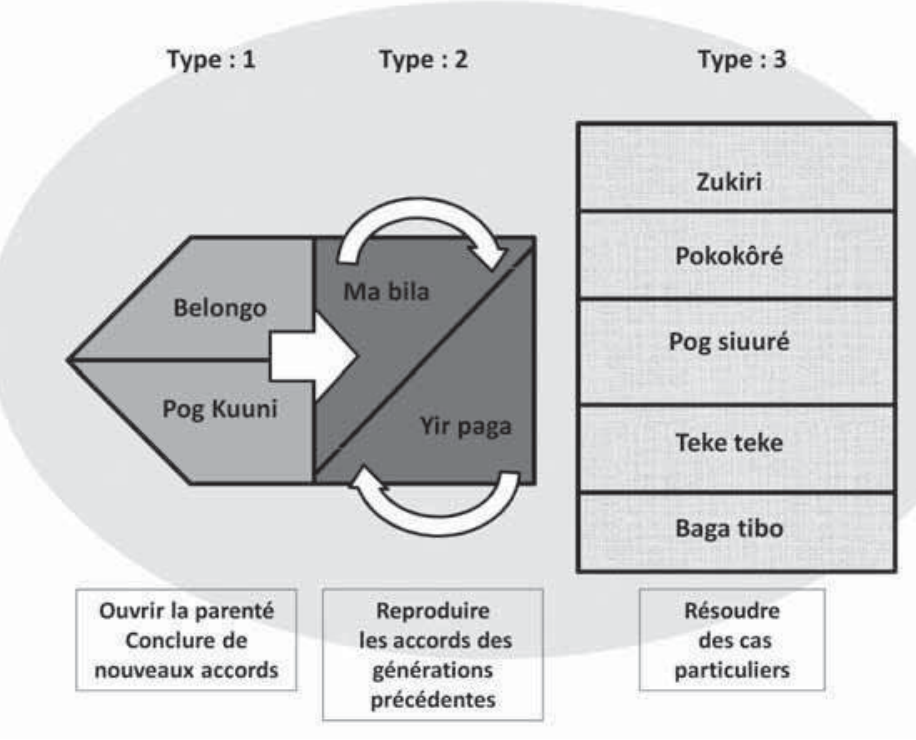

2 - Système de mariages mossi

17. Les hommes accèdent tardivement au mariage, parfois bien au-delà de vingt ans, alors que les femmes sont généralement mariées à dix-sept ans.

18. Pour un développement de cette argumentation, cf. Pierre-Joseph Laurent (2010). 
Le décor ainsi posé, il est à présent possible d'examiner de façon plus détaillée le fonctionnement de ces alliances.

- Alliances du premier groupe : ouvrir la parenté

Partons du fait que, dans la société mossi, le belongo (litt.: "chercher les faveurs de ") consiste, pour un père ou un grand-père (dans le sens de buudu kasma: "chef de segment de lignage"), à négocier auprès d'un homme d'un autre lignage l'obtention d'une épouse pour lui, ses collatéraux ou ses descendants. Détenant ainsi l'autorité en matière d'alliance, les aînés, le plus souvent des hommes (mais pas uniquement, nous le verrons), déterminent, selon les règles, mais aussi en fonction de leurs intérêts (personnels ou politiques), les fiancées autorisées. Le choix étant fixé, il s'ensuit de longues étapes.

L'alliance pog belongo est hasardeuse : elle peut se solder, après des années de négociation, par un échec. Cette négociation, longue et difficile, dépend essentiellement de la volonté des pères (des aînés) qui vivent souvent une relation conflictuelle avec leur premier fils et qui rechignent, dans ce cas, à leur trouver rapidement une épouse. Lorsque l'accord est finalement conclu, devant témoins et devant les ancêtres, il équivaut à un pacte transmissible de génération en génération.

La conclusion d'une alliance par belongo est donc particulièrement exigeante et aléatoire, si bien que les protagonistes n'y recourent que par nécessité. Elle repose sur de nouveaux rapports de confiance entre les lignages (basêm-yam), les générations et les sexes ${ }^{19}$, qui ne s'établissent que progressivement, après de longues prestations (reemdo) inhérentes aux obligations du fiancé :

LAZARE ( 55 ans, quartier de Bissiga, Kulkinka) : «Il fallait de dix à vingt ans pour gagner une femme par belongo. C'est à partir du moment où la fille a 7 ans que tu commences à aider sa famille».

Parmi l'ensemble d'obligations et de rituels requis pour un mariage par belongo, trois étapes viennent plus particulièrement ponctuer cette longue approche: le sakre teedo, le pog-puusem et le gam peogo. Le sakre teedo («donner du matériel à la fille») est la première obligation du fiancé, par laquelle il offre des présents à la jeune fille qu'il convoite. Cette étape constitue l'annonce officielle de la perspective d'une alliance ${ }^{20}$.

19. J'ai décrit ailleurs en détail les étapes de ce type d'alliance. Compte tenu de la complexité des étapes et des rituels mobilisés, il ne peut en être question ici (cf. Laurent 2009).

20. Durant toute cette phase, il est fréquent qu'un ou plusieurs membres du lignage demandeur viennent rendre des services dans les champs du lignage donneur. 
Le pog-puusem (salutations du lignage preneur au lignage donateur) est une autre étape décisive, demandant plusieurs semaines de préparation. Le déplacement des représentants du lignage demandeur dans le village donneur, en vue de concrétiser les relations de confiance (basêm-yam), en représente l'épisode crucial. Après de longues négociations, concernant la répartition au plus juste des présents apportés par les visiteurs et la manière de s'adresser aux ancêtres convoqués pour l'occasion, la délégation salue chaque membre du patrilignage donneur d'épouse. Le projet d'alliance est longuement détaillé. Les ancêtres sont consultés pour s'assurer qu'il respecte les interdictions; avant que l'accord ne soit définitivement scellé, des sacrifices sont effectués pour obtenir leur aval. Une issue favorable du pog-puusem mène à la dernière étape : la préparation, par les agnats de la jeune fille, du gam peogo ("panier entouré d'un filet ») ${ }^{21}$, qui rassemble les ustensiles de cuisine indispensables à la jeune épouse lorsqu'elle aura rejoint la cour de son mari. Finalement, le mariage se déroule à une date convenue par les parties. Un cortège, composé des agnats du fiancé et de ses amis, simule l'enlèvement de la jeune épouse (pog peegre), en demandant à son buudu kasma ("chef du lignage") l'autorisation de l'emmener et de la conduire dans le village de son mari. Ce jour du mariage (sibri daaré) est plutôt un jour triste, qui se passe sans grandes réjouissances pour la jeune fille et dans la crainte, pour la famille de son mari, qu'elle ne s'enfuie du domicile conjugal.

L'autre alliance du premier groupe, l'alliance par pog kuuni ("don d'une femme») differe du belongo dans la mesure où les phases de négociation n'existent pas: l'épouse est donnée à un ami de son père en guise de remerciement, pour sceller leur amitié. Les critères qui permettent de la classer dans ce premier groupe demeurent l'ouverture à de nouveaux lignages donateurs d'épouses et le fait qu'elle est susceptible d'être renouvelée (ré-enchaînée) à la génération suivante.

Face à ces exigences et contraintes inhérentes aux alliances du premier groupe, les Mossi retirent le sentiment que les femmes sont rares et les considèrent comme le bien le plus précieux qu'un homme puisse acquérir. Il semble également qu'ils cherchent à privilégier les alliances du deuxième groupe, qui reproduisent des accords déjà conclus et sont donc beaucoup plus simples à mettre en œuvre.

\section{- Alliances du deuxième groupe : reproduire les accords préexistants}

Les alliances du deuxième groupe revêtent deux formes. La première consiste à remplacer une épouse âgée par une jeune femme (yir paga:

21. Également désigné par le terme pag teedo («matériel de la femme»). 
"femme de la cour de la femme»), le plus souvent négociée par cette même épouse âgée (pubdba: "sœur de frère ») auprès de son patrilignage, pour lui venir en aide dans les tâches quotidiennes. La seconde forme est l'alliance ma bila ("petite maman»), au cours de laquelle un neveu négocie auprès d'un oncle maternel une de ses filles (ou une des filles de ses filles) - une cousine croisée matrilatérale - pour remplacer sa mère défunte. Les alliances de ce deuxième groupe sont donc beaucoup plus aisées à conclure que celles du premier: les longues étapes du belongo (sakre teedo, pog-puusem et gam peogo) n'ont pas lieu d'être puisqu'elles ont déjà été effectuées, une fois pour toutes, lors des alliances antérieures, c'est-à-dire à l'occasion d'un premier belongo. Les Mossi expliquent que ces étapes sont devenues inutiles car l'amitié existe déjà entre les lignages ainsi alliés :

Charles (65 ans, quartier de Bissiga, Kulkinka) : "Tu comprends, la parenté grâce à ces alliances-là (ma bila) ne finit jamais [...]. Attention, le mari ne doit pas saluer "ses beaux" comme dans le belongo. Il ne doit pas faire de salutations (pog-puusem) [...]. Moi-même, je n'ai rien fait [pas de salutations] pour l'obtenir ma bila, comme la parenté existe déjà ».

En 1990, je fus témoin à Kulkinka du décès de la mère de Charles. À l'époque, à près de soixante ans, il en fut fortement affecté. Quelques années plus tard, en novembre 1995, à l'occasion d'une visite, il me présenta ma bila, sa petite maman de huit ans. Il m'expliqua qu'elle n'était pas venue à la suite de belongo ni de pog siuuré, mais que c'est son oncle maternel (yaseba) qui lui avait donné ma bila. J'exposerai ici quelques éléments de cette histoire, particulièrement représentative de ces alliances du deuxième groupe et donc, plus largement, du système de pensée mossi et de sa conception de la parenté.

La mère de Charles, Sita, était originaire de Tanlili, un village situé à près de quatre-vingts kilomètres de Kulkinka. Le yaseba, l'oncle maternel de Charles, avait donné sa sœur à un homme de Kulkinka (un oncle paternel de Charles). Cet homme mourut et, selon la règle du lévirat, sa femme fut attribuée à un frère cadet du mari défunt, Sotissé, le père de Charles. Sotissé avait déjà une épouse et Sita eut trois enfants. Un jour, l'oncle maternel (yaseba) de Charles, le frère de Sita, lui réclama une de ses filles pour la donner à un $\mathrm{ami}^{22}$. Sotissé refusa, argumentant que sa femme venait de loin et qu'elle n'avait pas de famille pour l'aider à Kulkinka (une femme reste toujours une étrangère dans le lignage de

22. Sur la captation d'enfant, ici par les grands-parents maternels, cf. Suzanne Lallemand (1977: 211-213, voir aussi 1993: 151-152). 
son mari). Une longue brouille s'ensuivit. Et Charles, encore enfant, ne se rendit jamais à Tanlili avant 1992, soit deux ans après le décès de sa mère, Sita. Il nourrissait alors le désir de connaître sa famille utérine ${ }^{23}$ :

CHARLES : "Tanlili est un grand village. J'ai découvert de nombreux parents de ma maman. Ce jour-là j'ai beaucoup pleuré. Je pensais aux raisons qui m’avaient empêché de venir ici à l'époque où ma maman vivait».

Le neveu (yagenga), qui n'avait jamais rencontré son yaseba, lui rendit finalement visite dans l'intention de lui demander qu'une enfant de Tanlili vienne vivre avec lui et devienne ma bila. Il rappela à l'oncle les événements qui conduisirent leur famille à s'ignorer, mais, à présent que sa mère était morte, il le priait de rétablir les relations de parenté (rôgom). Charles expliqua à son yaseba qu'à la suite de leur différend, il avait été obligé de se présenter devant le chef mossi (le Chef du royaume du Zitenga), afin d'obtenir une fille pour remplacer sa mère défunte, étant donné que celle-ci n'avait pu demander elle-même à son propre lignage, selon l'usage, une yir paga pour l'aider dans les tâches quotidiennes qu'elle ne pouvait plus accomplir seule. Charles expliqua encore au vieil oncle qu'il voulait une "vraie maman" qui resterait dans sa cour. Aussi précisa-t-il : "Lorsque je la verrai, ce sera comme si ma maman vivait encore ». Le yaseba, touché par ses paroles, répondit à son yagenga d'aller voir ses filles et de choisir, parmi leurs propres filles, laquelle remplacerait sa mère. En 1995, une délégation de Kulkinka ramena ma bila, alors âgée de huit ans, chez Charles, c'est-à-dire chez son «fils " selon la terminologie mossi, non sans avoir été préalablement présentée à sa grande famille (buudu):

CHARLES: "Tous les jours, lorsque je la regarde, je pense à ma maman. Elle est petite, mais c'est elle qui la remplace, je l'appelle ma bila: c'est ça le remplacement. Je l'appelle ma bila, petite maman ou pog nyanga, la vieille. Elle me rappelle sans cesse ma maman. Pour m’amuser, je lui demande de téter son sein, mais elle refuse...».

Le cas de Charles correspond donc à l'exemple parfait d'alliance ma bila : après le décès de sa mère, un fils devient demandeur d'épouses auprès de ses oncles maternels. Il justifie cette revendication à la fois par le chagrin que lui procure la disparition de sa mère, par le fait que plus personne ne se rendrait dans le village maternel si la parenté s'interrompait et par la croyance que, via la transsubstantiation partielle (réincarnation partielle d'un ancêtre), il pourra retrouver sa maman. Il ne peut pas l'épouser car,

23. Pour plus de détails concernant ce cas qui nous plonge au cœur de la parenté moaga et de son système de pensée, cf. Pierre-Joseph Laurent (2009). 
dans le système de pensée mossi, bien exprimé par la terminologie, cette fille équivaut à sa maman : il l'appelle "mère » et elle l'appelle "fils ", alors qu'ils ont le même âge, voire, comme dans notre exemple, deux générations d'écart. Mais, par cet acte, lui-même devient un donneur d'épouses, soit pour un fils d'une co-épouse de son père, soit pour un fils du frère de son père (un cousin parallèle patrilinéaire) ou, plus largement, pour un membre de son patrilignage. La terminologie mossi oriente les comportements entre les personnes et donne des indications sur les mariages possibles, voire souhaitables, sans pour autant prescrire qui épouser.

Dans les alliances de ce deuxième groupe, yir paga et ma bila sont donc des cousines croisées matrilatérales et portent le nom de ma ("maman») pour Ego dans la terminologie mossi. Ces alliances, selon l'idéologie patrilinéaire mossi, n'exigent plus la mobilisation d'un chef de segment du lignage, mais, plus modestement, l'intervention d'une mère (pour la yir paga) ou d'un neveu maternel (dans le cas de ma bila). Elles sont fréquentes et les plus valorisées; tant qu'elles ne sont pas interrompues par des disputes, elles permettent de poursuivre et de reproduire, de génération en génération, les relations de parenté, initiées par les aînés (les pères) à la suite d'un belongo, voire d'un pog kuuni. C'est dans ce sens que je propose de comprendre et d'intégrer ici les analyses de Virginie Vinel sur le « ré-enchaînement des alliances ${ }^{24}$.

\section{- Alliances du troisième groupe : résoudre des problèmes particuliers}

Le troisième groupe d'alliances existant dans le système de parenté mossi (sukiri, pokokôré, pog siuuré, teke teke, baga tibo) ${ }^{25}$ s'apparente plutôt à des alliances $a d$ hoc qui répondent à des problèmes spécifiques. Il s'agit surtout de répondre à des besoins du groupe, ou encore à des problèmes de stérilité, de fuite d'une épouse ou de décès..., en permettant aux hommes et femmes qui y sont confrontés de trouver, malgré tout, un conjoint que l'on sait indispensable pour l'adulte mossi. Une description

24. Les ré-enchaînements d'alliance, la réciprocité entre lignages et la circulation des fiancées dans la parenté permettent la perpétuation d'échanges entre un nombre restreint de lignages et le maintien de l'endogamie locale (Vinel 2005 : 211). Au-delà du fait qu'il convient de coupler cette endogamie à une ouverture, l'auteure met en lumière trois types de ré-enchainements d'alliance: un homme peut recevoir plusieurs épouses d'un même lignage; les frères réels ou classificatoires obtiennent des épouses d'un même lignage; un homme prend une épouse dans le lignage d'une co-épouse de sa mère ou dans le lignage d'une co-épouse de sa grand-mère. J'ai déjà indiqué que si ses informations sont très précieuses, elles restaient à intégrer dans une proposition générale d'approche du système de mariages mossi et de la terminologie omaha. Par une autre entrée, Doris Bonnet a déjà rendu compte de ce processus, lorsqu'elle traite des droits de l'enfant de la donatrice (ici la pugbda: «sour de père ») sur le premier enfant de la yir paga dont elle avait permis le mariage dans le lignage où elle est elle-même mariée (1988: 54).

25. Pour une description détaillée de l'ensemble de ces alliances, cf. Pierre-Joseph Laurent (2010). 
détaillée de ces alliances ne peut malheureusement prendre place ici tant les questions qui les caractérisent sont techniques, parfois complexes. C'est le cas, notamment, des alliances par pog siuuré26 ("don différé d'épouse »), plutôt réalisées entre lignages d'ascendance royale (cf. Marc-Éric Gruénais 1979: 41-42); ou encore, de celles par teke teke, qui correspondent au don réciproque de femmes, en situation de défiance par exemple.

\begin{tabular}{|c|c|c|c|}
\hline Auteurs & $\begin{array}{c}\text { Alliances de type I } \\
\text { Ourrir }\end{array}$ & $\begin{array}{c}\text { Alliances de type } 2 \\
\text { Reproduire }\end{array}$ & $\begin{array}{c}\text { Alliances de type } 3 \\
\text { Résoudre des cas } \\
\text { particuliers }\end{array}$ \\
\hline \multirow{2}{*}{$\begin{array}{c}\text { Doris Bonnet } \\
\text { sur } 100 \text { mariages } \\
\text { observés } \\
(1988: 53-54)\end{array}$} & $\begin{array}{l}\text { Belongo: } 39 \\
\text { Pog kuuni: } 7\end{array}$ & $\begin{array}{l}\text { Yir paga: } 28 \\
\text { Ma bila: } 7\end{array}$ & $\begin{array}{l}\text { Fuite, vol.... : } 8 \\
\text { Pog siuuré: } 10 \\
\text { Autres : I }\end{array}$ \\
\hline & Total : 46 , soit $46 \%$ & Total : 35 , soit $35 \%$ & Total : 19, soit $19 \%$ \\
\hline \multirow[t]{2}{*}{$\begin{array}{c}\text { Virginie Vinel } \\
\text { sur } 45 \text { mariages } \\
\text { observés } \\
(2005: 65)\end{array}$} & Belongo: 9 & $\begin{array}{c}\text { Yir paga et Ma bila: } \\
21\end{array}$ & $\begin{array}{l}\text { Pog siuuré : } 1 \\
\text { Lévirat : } 3 \\
\text { Fuite, vol : } 6 \\
\text { Non connu : } 5\end{array}$ \\
\hline & Total : 9 , soit $20 \%$ & Total : 21 , soit $46,7 \%$ & Total : 15 , soit $33,3 \%$ \\
\hline
\end{tabular}

Distribution des alliances mossi selon les trois types

\section{Idéologie du patrilignage et univers avunculaire}

Outre les alliances du troisième groupe qui, pour importantes qu'elles sont, restent à part, nous avons montré que les alliances du deuxième groupe, yir paga et ma bila, généralement les plus nombreuses, s'articulent avec celles du premier groupe, belongo et pog kuuni, qu'elles reproduisent. Ainsi, tant que des différends (tels que la rupture avec l'oncle maternel évoqué dans notre exemple, ci-dessus) ne viennent pas interrompre la relation, les alliances du deuxième groupe peuvent se ré-enchaîner génération après génération ${ }^{27}$.

26. Pog suuiré: de pog, paga («femme») et siubo («donner dans l'attente d'un profit à retirer»), cf. le Père Gustave Alexandre (1953). Pog siuuré : "pratique consistant à rendre le premier enfant au donateur de la mère", cf. Norbert Nikiéma \& Jules Kinda (1997 : 712).

27. Sachant que notre intention n'est en aucun cas d'élever ce type d'alliance - celle où la cousine croisée matrilinéaire est donnée par Ego à un cousin parallèle patrilinéaire - au rang de mariage canonique chez les Mossi. Répétons-le une fois encore, chaque alliance importe et remplit son rôle dans ce système, dans la mesure où elle est mobilisée en fonction du contexte et des circonstances. 
L'alliance ne regarde pas seulement les individus concernés, mais tout un segment de lignage; pas une, mais plusieurs générations. De sorte que les alliances dépendent tout autant de l'autorité des aînés (hommes et femmes) du lignage que des mères et leurs fils, en fonction de leurs capacités de négociation, de leurs réputations, mais également du potentiel d'alliances héritées des générations précédentes et devant être négociées, c'est-à-dire réactivées à chaque génération pour les rendre effectives. L'hypothèse défendue dans ce texte est que la terminologie de parenté mossi concourt à rendre possible cette effectivité, en forgeant et valorisant dès le plus jeune âge les conduites ad hoc, celles aptes à opérer ce renouvellement des alliances conclues par les générations antérieures. Autrement dit, un pacte d'alliance conclu par ces dernières n'a de valeur que tant qu'il est actualisé par le témoignage d'un comportement adéquat, particulièrement attentif aux droits et aux devoirs de chacun, de la part de la génération suivante. Un pacte d'alliance n'entraîne pas d'obligations fermes, mais des potentialités activées notamment par la terminologie. C'est dans ce sens qu'on peut parler de structure semi-complexe de la parenté, dans la mesure où elle n'est pas contraignante mais indicative, là où la terminologie n'indique pas vraiment avec qui se marier, mais contribue plutôt à orienter les conduites afin de permettre l'actualisation des potentialités contenues dans les pactes hérités.

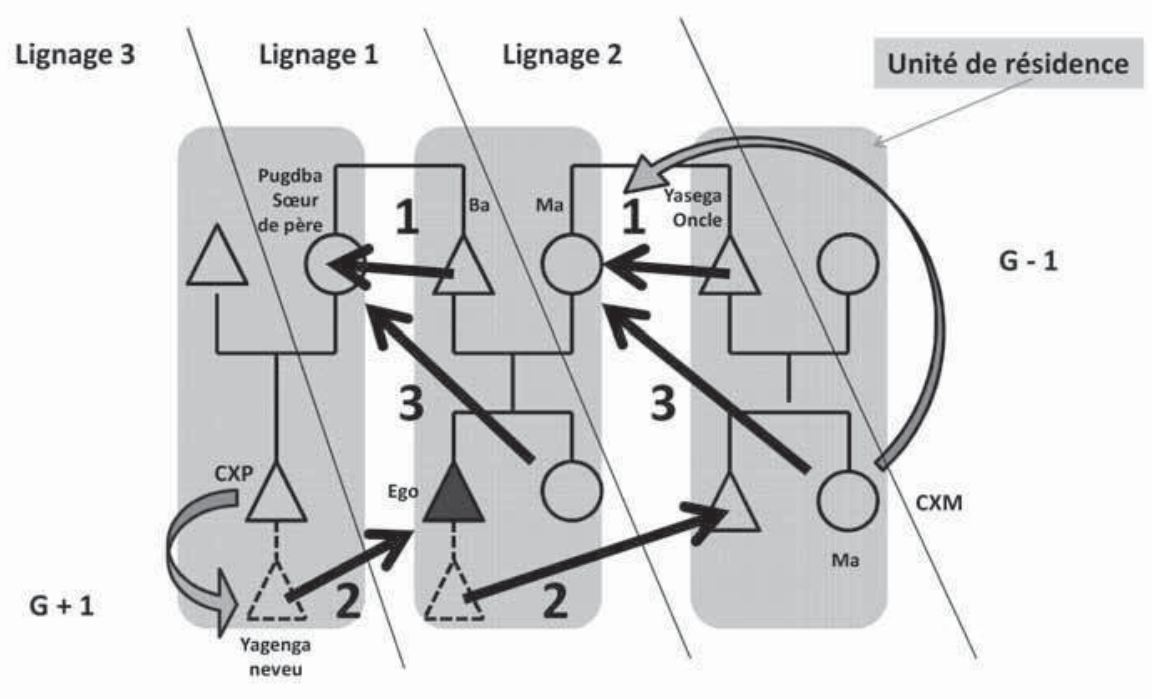

3 - Envoyer (I), renvoyer (2) et remplacer (3) 


\section{Envoyer : la femme est paga ("épouse") et pugdba ("sœur de père")}

Quand une jeune épouse (paga) arrive dans la famille de son mari (sida), elle occupe une place au plus bas de l'échelle hiérarchique. Lors de son mariage, quelle que soit la forme de l'alliance, elle doit quitter son patrilignage et son village natal pour rejoindre la cour (unité de résidence) de son mari, où elle restera toute sa vie une étrangère [Fig. 3, point I voir supra]. La société mossi étant patrilinéaire, ses enfants appartiendront au lignage de son mari ; et s'il lui arrivait de rompre son mariage (ce qui était assez fréquent dans le village où j'ai mené mes enquêtes), pour fuir par exemple avec un amant, elle partira en laissant ses enfants au lignage de "ses maris" (suivant la terminologie classificatoire). La société mossi est par ailleurs très hiérarchisée et les relations au sein du patrilignage sont strictement organisées autour des relations aînés/cadets, hommes/femmes, consanguins/alliés.

La jeune femme peut arriver en tant que première épouse ou en tant que co-épouse (pugto), et ce n'est que progressivement qu'elle parviendra à faire sa place dans le lignage de son mari. Pour ce faire, de multiples possibilités s'offrent à elle, au-delà de la jeunesse et de l'attrait physique qui peuvent entraîner des jalousies tenaces, l'habilité dans le travail (agriculture, élevage, artisanat) et, surtout, la naissance de ses premiers enfants y contribueront car, puisqu'elle reste une étrangère là où elle est mariée, ses enfants deviendront ses principaux alliés. Par ailleurs, avec le temps, elle pourra devenir la mère éducatrice ( $m a$ wubduga) des enfants d'épouses plus jeunes, avec un droit d'autorité sur eux et sur leurs géniteurs (Lallemand 1977 : 190 sqq.). Elle augmentera encore son autorité lorsque, tirant parti de son statut de sœur (pugdba), elle demandera auprès de son patrilignage une jeune fille pour venir la remplacer auprès de son mari, qui, lui, pourra donner cette jeune fille à son frère cadet (il s'agit ici de yir paga). À ce niveau, il est important de relever que la première fille issue de cette union revient à l'enfant de la donatrice : pugdba transforme ainsi son premier enfant en donneur d'épouse pour son lignage. Doris Bonnet précise que si cet enfant de la donatrice est un garçon, celui-ci pourra donner la fille en question à un lignage tiers, et si cet enfant est une fille, celle-ci, soit la fille pugdba et de yir paga pourront devenir des co-épouses (pugto) d'un homme appartenant à un lignage tiers (Bonnet 1988: 54).

Pour résumer, une épouse renforce progressivement sa position dans le lignage de son mari et confere à son fils la capacité d'accéder à des filles qu'il pourra à son tour donner en mariage. Progressivement donc, l'épouse la plus âgée occupe une place de plus en plus enviable dans la cour de son mari (sida), soit en sa qualité de sœur via l'accès à des épouses potentielles dans 
son patrilignage, soit en sa qualité de mère génitrice via la place occupée par ses propres enfants dans le lignage de son mari, soit en sa qualité de mère éducatrice ayant un droit d'autorité sur les enfants de jeunes coépouses.

Paga dans la famille de son mari, elle est pugdba pour Ego, c'est-à-dire une "sœur de père " qui vit au loin chez ses maris et qui revient de temps en temps rendre visite à son père et à ses frères. Lors de ces visites dans son village natal, à l'occasion de la naissance d'un enfant ou de funérailles par exemple, elle se montre impérieuse envers les épouses de ses frères et envers leurs enfants, pour lesquels elle participe de l'autorité du patrilignage : elle est généralement crainte et ses paroles sont écoutées avec respect (même si parfois, dans son dos, les jeunes de la cour se jouent d'elle). Notamment, en tant que pugdba, elle fait savoir aux jeunes épouses de ses frères que leurs enfants lui appartiennent, en leur rappelant qu'elles sont étrangères dans cette cour et que c'est elle qui détient l'autorité sur leurs enfants puisque c'est elle qui pourra les marier.

Cet ascendant est manifeste dans plusieurs rituels décrits par Doris Bonnet. Ainsi, lors d'un accouchement, pugdba dirigera le rituel lié à l'enterrement du placenta, qui représente le double de l'enfant ${ }^{28}$, pour montrer que le nouveau-né est déjà considéré comme dépendant de l'autorité du lignage de son père. C'est encore pugdba qui séparera progressivement la mère de l'enfant à l'occasion du sevrage, et qui rappellera à la mère que, si elle venait à fuir, elle laisserait l'enfant à ses maris (Bonnet 1988 : 45). C'est aussi généralement pugdba qui est apte à déterminer le segre (partie d'un ancêtre) et le sondre (nom) de l'ancêtre en ligne agnatique revenu dans la personne du nouveau-né (Ibid. : 88) ${ }^{29}$.

La sœur tient également un rôle déterminant dans l'organisation des funérailles au sein de son patrilignage, dans l'éducation des enfants des épouses de ses frères, ou encore en cas de maladie. En somme, si une femme, en tant que paga (" épouse»), reste une étrangère longtemps située au bas de la hiérarchie dans le lignage de son mari, elle se voit, en tant que sœur et tante paternelle (pugdba), accorder un crédit tout différent. Les membres de son patrilignage et leurs épouses lui doivent le respect et sont tenus de s'adresser à elle avec déférence : "La tante c'est comme un père sauf que c'est une femme ", relève Doris Bonnet, associant ainsi la sœur à un personnage masculin par ses pouvoirs (Ibid.: 52).

28. Le placenta (naaba) est le siège de segre, la part d'ancêtre appelée à revenir.

29. À ce propos, il est intéressant de noter que la femme, par sa double appartenance lignagère - épouse de son mari et sœur pour son lignage paternel -, peut revenir (pour reprendre la formule utilisée par les villageois) comme consanguine et alliée. Notons encore que certaines personnes peuvent revenir, en même temps, plusieurs fois, sachant qu'il est question chez les Mossi d'une transmission transgénérationnelle de principes vitaux. 
Lorsqu'une jeune épouse est envoyée par son patrilignage chez son mari, la famille de celui-ci a ainsi contracté une dette envers celle de sa femme: "Ils ont perdu quelqu'un ", disent les Mossi. Comme nous allons à présent le voir, c'est précisément ce qu'indique clairement la terminologie mossi lorsque, pour Ego, le fils de la fille donnée est désigné par le terme yagenga ( "neveu »), c'est-à-dire comme un cousin croisé patrilatéral ainsi descendu d'une génération.

\section{Renvoyer : le yagenga}

Suzanne Lallemand a montré que confier un enfant est une pratique courante dans la société mossi (1993: 55-56, 80-81, 93-94 et 150-152). Cet acte prend des formes multiples. À l'intérieur du patrilignage, une aînée peut "capturer " l'enfant d'une jeune épouse et devenir son éducatrice. À l'extérieur, la pugdba peut, à sa demande explicite, se voir confier l'enfant d'un frère, ou encore le lignage maternel peut réclamer au mari de la sœur un de ses enfants. Signalons enfin que, d'une certaine manière, la yir paga et la ma bila sont des enfants confiées, dans la mesure où ce sont des petites filles destinées à devenir des épouses dans un autre lignage.

Parmi ces cas, celui où un neveu maternel est confié à ses oncles (terminologie classificatoire) est fréquent. Dès l'introduction, nous indiquions qu'un homme, en sa qualité de neveu maternel, existe d'abord en ce qu'il représente, pour son oncle, le remplacement de sa mère. C'est ce que nous allons à présent développer.

Alors que la sœur (pugdba) a été envoyée vivre dans l'unité de résidence de son mari, en retour, elle "renvoie" un fils pour aider son patrilignage. Autrement dit, pour le lignage donneur d'épouse, le yagenga ("neveu») remplace, "vient" à la place de la pugdba. Toutefois, pour qu'il puisse partir (ici dans le sens morré de "revenir ») sans perturber l'ordre hiérarchique de son lignage maternel, la terminologie mossi le descend d'une génération : ce n’est pas tant un cousin croisé patrilinéaire qui vient aider le lignage de sa mère, mais bien un neveu pour Ego, masculin dans le raisonnement. Les cousins croisés basculent donc autour d'Ego et ce processus indique qui doit venir en remplacement et sous quel statut [Fig. 3, pt. 2 voir supra]. Les Mossi disent à ce propos : "Ta maman n’est pas là, mais toi son fils tu es là, chez tes oncles maternels »; "Le fils vient remplacer sa mère qui est partie "; "En donnant une fille, on ne perd pas une force de travail puisqu'elle nous renvoie un homme"; "Celui qui a reçu notre sœur est le bénéficiaire de la femme, mais nous, nous sommes les oncles, alors nous sommes les bénéficiaires de son fils ». Dans ce cas, formellement, une femme n'est pas échangée contre une autre femme, mais bien contre un homme! 
Dans cet espace ouvert de la savane arborée, il faut comprendre que, historiquement, il était de la plus haute importance pour les villages mossi de pouvoir compter sur un maximum d'hommes, pour cultiver ou pour se défendre tant des animaux sauvages que des ennemis. C'est dans ce sens qu'un neveu maternel est toujours bien accueilli chez ses oncles. Il est dit qu'il vient renforcer le village ${ }^{30}$.

Généralement, le yagenga ne vit pas chez son oncle maternel (yaseba); il réside plutôt dans son patrilignage, où il cultive les terres du lignage. Il peut toutefois résider chez son oncle, avons-nous expliqué, en tant qu'enfant confié, ou alors, plus âgé, il peut choisir de s'y installer. Dans ce cas, bien qu'il soit étranger, il sera le bienvenu, car, pour les raisons que nous venons d'évoquer, on ne refuse jamais de terres à un yagenga. C'est en ce sens que je qualifie la société mossi de société patrilocale, certes, mais avec une tendance avunculocale.

La relation yagenga/yaseba est donc importante. Elle inverse en quelque sorte les relations hiérarchiques du patrilignage, empreintes d'autorité et de respect : alors que le fils traite son père, ainsi que les autres aîné(e)s de son patrilignage, avec déférence et soumission, il trouve auprès de ses utérins une relation de respect mutuel, de confiance et d'assistance. Le yagenga tient, en outre, un rôle de médiateur et de pacificateur au sein du lignage de son yaseba du fait qu'il ne peut être considéré comme un rival. Il est effectivement un étranger pour ses oncles puisqu'il appartient au lignage de son père et, au regard de la terminologie, il se trouve dans une position d'infériorité, puisqu'il n'est pas un cousin mais un neveu pour son cousin croisé matrilatéral, ce qui lui permet de ne pas apparaître comme un concurrent potentiel dans l'ordre hiérarchique de son matrilignage. En tant qu'étranger dans la cour de ses oncles - tout comme sa mère l'est dans celle de son mari -, le neveu n'hérite pas de son oncle, ne peut épouser ses filles qui sont des mères pour lui, ni se remarier avec les femmes de cet oncle après son décès (Lallemand 1977: 331). En revanche, il peut prendre ce qu'il veut dans la cour de ses oncles sans que cela pose problème. C'est ainsi que, de par la dualité de sa position, le yagenga peut également être amené à jouer un rôle déterminant dans le lignage de ses maternels.

30. Lors de mon premier séjour à Kulkinka (de juillet 1988 à octobre 1991), quelques semaines après mon arrivée, je fus témoin d'un conflit - dramatique, puisqu'il y eut un mort - avec le village voisin de Tanghin au sujet de l'accès au point d'eau. Alors que, la veille, la rumeur courait que Kulkinka risquait d’être attaquée, je découvris, au matin, une véritable petite armée composée en grande partie de jeunes hommes que je ne connaissais pas. Je compris qu'il s'agissait de neveux maternels (yagense, pluriel de yagenga) qui avaient convergé durant la nuit vers le village de leurs oncles pour leur prêter main-forte. 
Il est dit que, à travers le neveu, l'oncle voit sa sœur. Entre ces personnes, une relation de confiance peut donc s'établir, qualifiée de tog sida, "parole juste ", de sorte que l'oncle n'a pas d'autorité sur lui. Suzanne Lallemand a montré, qu'avec le temps, le neveu s'autocensure : il cesse de prendre tout ce qu'il désire dans la cour de son oncle, devenant solidaire des biens de son matrilignage et un de leurs meilleurs gardiens (Ibid.: 332). Progressivement, il assume aussi un rôle d'intermédiaire: par exemple, en cas de conflit, il est suffisamment proche pour recevoir les confidences, et suffisamment distant pour écouter les différents points de vue et trancher en fonction de l'intérêt général du lignage. Grâce à sa position de fils de sœur, il est le détenteur et le maître du pardon; il peut punir des fautifs à la place du chef de la cour. Ou encore, lorsque les circonstances l'imposent, il peut tenir un rôle central dans les rituels en y remplaçant son oncle, ce qui lui donne des prérogatives supérieures à celles d'un fils.

Suzanne Lallemand a en outre souligné que cette position ambivalente du neveu lui permet d'apporter la paix, l'entente et le consensus au sein du lignage de son oncle maternel; elle lui confere un statut de faiseur de paix (zemes taaba). En tant que fils de la fille du lignage, il est respecté et sa parole écoutée, mais sur le mode de la familiarité et des relations à plaisanteries (rakiya) - notamment avec les épouses de ses oncles. Cette ambivalence est sans conteste la raison de son excellente intégration (Ibid. : 346). Il existe donc un transfert de compétences entre l'oncle et le neveu, faisant coexister l'idéologie de patrilignage, autoritaire et très hiérarchisée, avec l'univers avunculaire fait de complicité et de tempérance.

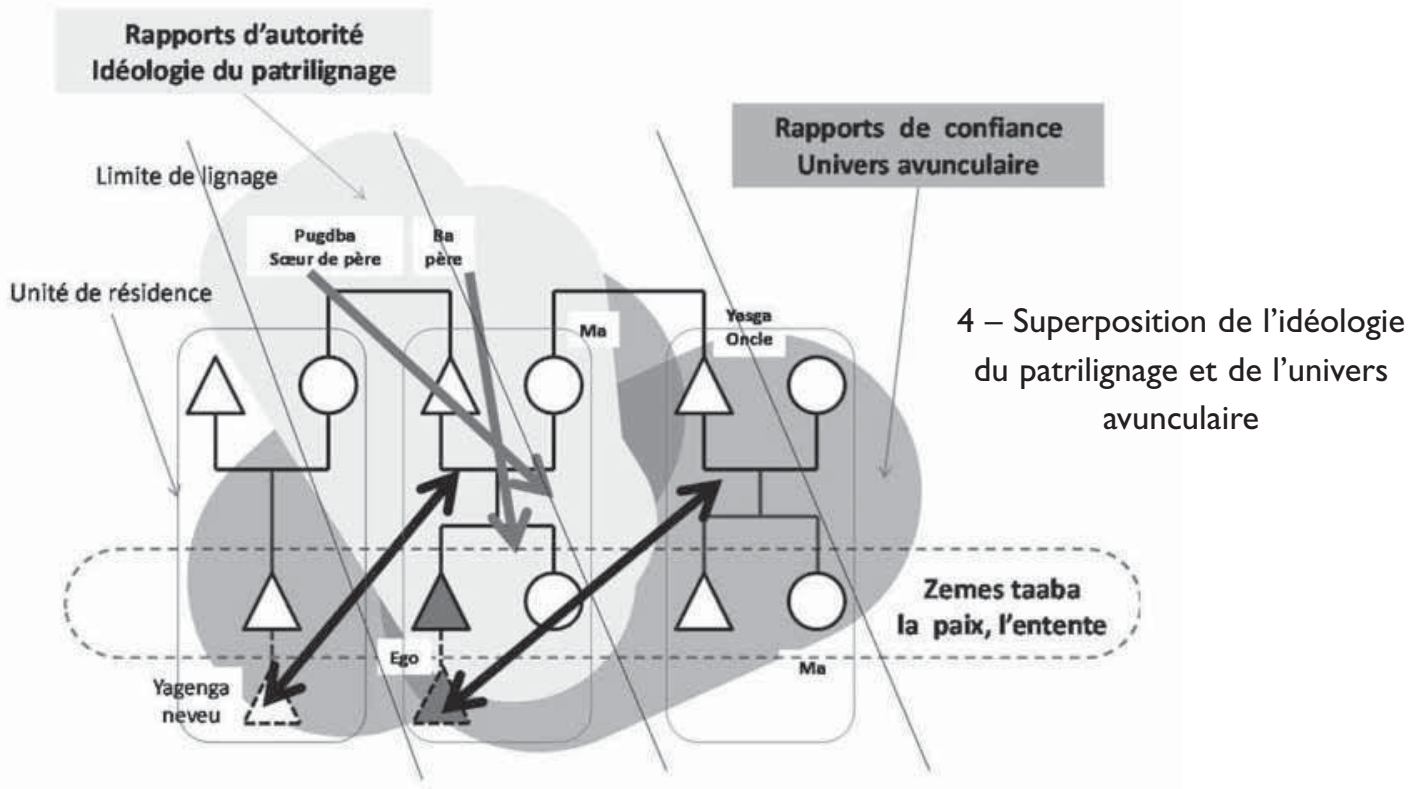


Si un neveu veille à la cohésion interne de son matrilignage, s’il surveille, parfois avec véhémence, les alliées de ses oncles, il peut également prétendre à l'engagement matrimonial des filles du lignage maternel ( $m a$ bila, voire yir paga $)^{31}$. C'est dans ce sens qu'il convient de comprendre que le basculement oblique autour d'Ego oriente les comportements pour, finalement, définir qui vient en remplacement de qui dans la succession des générations.

\section{Remplacer : yir paga ou ma bila}

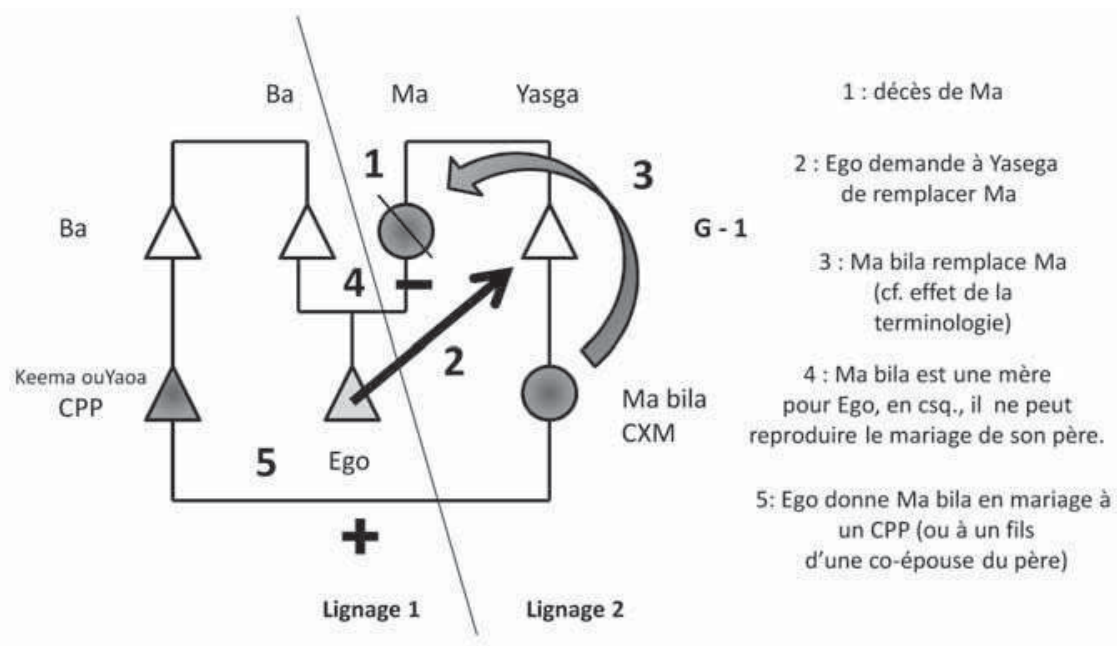

5 - Remplacer : yir paga ou ma bila

Dans cette alliance que nous avons déjà décrite, dite du deuxième groupe, un autre effet important de la terminologie mossi s'exprime clairement. À propos de l'alliance ma bila, rappelons qu'un neveu (yagenga) négocie auprès d'un oncle maternel (yasega) une de ses filles (ou une fille de ses filles) ${ }^{32}$ pour « remplacer» sa mère défunte [Fig. 5, pt. I]. Il justifie sa demande par le fait qu'il pourra ainsi retrouver sa mère à travers cette fille [Fig. 5, pt. 2]. Ma bila peut être confiée très jeune à la famille d'Ego (parfois vers 4 ou 5 ans), qui devient, avec une de ses épouses, son éducateur ( $m a$ et ba wubduga) [Fig. 5, pt. 3]. Dans sa famille d'accueil, Ego et ses enfants sont des fils et filles pour ma bila ${ }^{33}$. Ego et ses enfants ne peuvent dont pas épouser ma bila, leur petite maman, et reproduire ainsi le mariage de leurs

31. Cf., à ce propos, les développements particulièrement intéressants de Suzanne Lallemand (1977: 157, 330 et 332).

32. Elles sont toutes appelées ma par Ego, en raison de la terminologie classificatoire.

33. Dans la terminologie mossi, Ego appelle «mère» sa cousine croisée matrilatérale, et elle l'appelle fils. 
père et grand-père [Fig. 5, pt. 4]. Lorsqu'elle aura atteint l'âge de pouvoir être mariée (17 ans, le plus souvent), Ego donnera ma bila en mariage à un fils d'une co-épouse de son père, à un cousin parallèle patrilinéaire, ou encore à un membre plus éloigné de son patrilignage [Fig. 5, pt. 5]. Il est intéressant de souligner que la terminologie de parenté mossi renseigne sur le type d'exogamie en vigueur, notamment sur le fait que les matrilignages sont généralement prohibés (sur trois générations).

Ainsi donc, dans la continuité de "ses pères" qui avaient conclu un premier pacte d'alliance avec un lignage tiers, un fils peut devenir, par sa mère (soit de son vivant avec la yir paga, soit après son décès avec la ma bila), à la fois demandeur d'épouses auprès de ses oncles maternels et donneur d'épouses auprès de cousins parallèles patrilinéaires: l'oncle maternel donne une fille à son neveu qui la donne à son tour en mariage à un cousin parallèle patrilinéaire.

Dans un contexte où la recherche d'une épouse pour leurs fils est particulièrement difficile pour les aînés et où les hommes accèdent tardivement au mariage, la terminologie brosse les contours de ce que doivent être les conduites socialement acceptables, les droits et devoirs censés conduire au respect des pactes matrimoniaux conclus entre lignages par les générations précédentes. Ce ne sont pas d'obligations formelles qu'il s'agit, mais plutôt de comportements intériorisés dès le plus jeune âge, et induits soit par l'idéologie hiérarchique du patrilignage, soit, à l'opposé, par le climat d'assistance mutuelle de l'univers avunculaire. Ce processus repose, au départ, sur un pacte établi entre deux lignages inconnus (voir l'alliance par belongo) où, pour ce faire, tous les membres de chaque lignage ainsi que leurs ancêtres ont été pris à témoins (voir le pog-puusem), afin d'établir le bien-fondé de cette alliance et, bien au-delà, afin qu'elle puisse être transmissible de génération en génération, c'est-à-dire tant que lesdits comportements sont respectés. Pour cela, ceux-ci reposent cette fois sur le basculement oblique autour d'Ego, indiquant qui vient en remplacement de qui pour maintenir, entre les lignages, les termes des accords passés : un yagenga est renvoyé pour remplacer sa mère et ma bila vient remplacer sa tante paternelle (pugdba) [Fig. 3, pt. 3 voir supra].

Les alliances mossi se concluent donc dans le temps long et étendent leurs effets sur plusieurs générations. Parfois, plus de cinquante ans se sont écoulés entre l'octroi d'une épouse et son remplacement, sans compter que ce simple remplacement peut lui-même être la conséquence d'un remplacement précédent... On comprend ainsi que les générations se chevauchent souvent entre les lignages preneurs et donneurs d'épouses, dans la mesure où un oncle peut aisément être plus jeune que son neveu. Peu importe, puisque la terminologie est classificatoire et que ce sont les termes 
d'adresse utilisés pour désigner une personne qui comptent davantage que l'identité de la personne elle-même: cette terminologie classificatoire combinée à la conception de la personne chez les Mossi (dans le sens d'une forme de permanence de l'être) contribue à faire en sorte que, à ce niveau du grand jeu des alliances, le rôle (pour ne pas dire les rôles) qu'il doit tenir transcende l'individu. De sorte qu'il soit jeune ou vieux, l'important est que, pour son neveu utérin, l'oncle remplisse son rôle dans toute la plénitude de la fonction.

Soulignons, enfin, que le demandeur d'épouse (Ego qui, ici, demande une fille à son oncle maternel) ne peut se marier avec la fille qu'il reçoit. En conséquence, l'interdit induit par la terminologie (un fils ne peut pas épouser sa mère) le transforme en donneur d'épouse pour son propre patrilignage. Par le basculement oblique autour d'Ego, cet interdit devient en quelque sorte un «interdit mobile", puisque rien n'empêche une autre personne du lignage d'Ego de se marier avec la fille ainsi reçue. Plus encore, puisque, grâce à son oncle maternel (yaseba), Ego peut se transformer en donneur d'épouses au même titre que ses aînés ${ }^{34}$, cela nuance et relativise le rapport aînés/cadets, ces derniers occupant une place plus influente dans leur patrilignage.

Dans cet article, nous nous sommes efforcés de rendre compte du mécanisme de fusion oblique caractéristique du système omaha, en appréhendant la terminologie de parenté mossi dans des contextes donnés, au plus près des pratiques et des représentations.

L'alliance chez les Mossi n'est jamais une affaire privée. Elle concerne avant tout le buudu («lignage»). C'est dans ce sens qu'un Mossi dira, qu'en dehors du système de parenté (rôgom), il n'est pas possible de trouver une épouse, et que sans la famille on n'est rien. Plus encore, même si le mariage concerne avant tout deux segments de lignages, pour en comprendre le fonctionnement et l'articuler à la terminologie de parenté, il importe de porter le regard sur au moins trois lignages et trois générations. Dans ce système, les pactes d'alliance concernant l'octroi d'épouses deviennent transmissibles de génération en génération, la génération actuelle bénéficiant des accords noués par les aînés - et au-delà, par les ancêtres -, qu'elle peut reproduire via les alliances du deuxième groupe. Or, la terminologie contribue à rendre effectif ce processus, en favorisant, dès le plus jeune âge, des conduites socialement valorisées, et en intervenant sur la manière de mettre en scène des comportements

34. Et pas seulement son père ou la sœur de père (pugdba). 
rituels, parfois ambivalents, réglant minutieusement les relations des uns avec les autres dans l'intention d'orienter les projets d'alliances possibles. Notamment, le principe de basculement oblique autour d'Ego indique qui vient en remplacement de qui et selon quel statut, afin de favoriser le respect des termes de ces accords passés entre les lignages.

Ainsi, pour comprendre ce système terminologique, a-t-il été nécessaire d'embrasser d'un seul regard cet ensemble d'alliances, de rester attentif aux perspectives transgénérationnelles et de tenir compte de divers éléments contextuels, dont la conception de la personne, mais également ceux concernant les relations d'autorité au sein du patrilignage et celles, plus équilibrées, de l'univers avunculaire. Enfin, cette approche de la terminologie mossi aura permis de montrer l'évolution, grâce à leurs rôles dans les alliances matrimoniales, du statut des femmes et du rapport aînés/cadets.

Pour terminer, insistons sur le fait que le yagenga tient le rôle de médiateur et de pacificateur au sein du lignage de son yaseba. Il tient ce rôle car il n'est pas un rival et car, en raison de l'ambivalence de sa position, il est un faiseur de paix (zemes taaba). Outre que cette société est de nature guerrière, un autre trait de la terminologie consiste donc à imposer une forme de paix (avunculaire), transversale aux unités de résidence et qualifiée de zemes taaba ("ajuster ensemble»).

MOTS CLÉS/KEYWORDS : Mossi - parenté/kinship - terminologie de parentélkinship terminology - mariage/marriage - système omaha/Omaha system - Burkina Faso. 
Alexandre, Gustave (Père)

1953 La Langue moore, I et II.

Dakar, IfAN («Mémoires de l'Institut français d'Afrique noire» 34).

\section{Badini, Amadé}

1979 «Les éléments de la personne humaine chez les Moose", Bulletin de l'IFAN, série B 41 (4) : 787-818.

\section{Bonnet, Doris}

1988 Corps biologique, corps social.

Procréation et maladies de l'enfant en pays mossi, Burkina Faso. Paris, Orstom

(«Mémoires » 110).

\section{Douglas, Mary}

1999 Comment pensent les institutions.

Trad. de l'anglais par Anne Abeillé.

Paris, La Découverte - MAUSS

(«Recherches. Bibliothèque du MaUss »).

\section{Godelier, Maurice}

2004 Métamorphoses de la parenté.

Paris, Fayard.

\section{Gruénais, Marc-Éric}

1979 "L'échange différé des femmes chez les Mossi ", L'Ethnographie. Revue de la Société d'ethnographie de Paris 79 (1) : 41-60.

1985 «Aînés, aînées, cadets, cadettes : les relations aînés/cadets chez les Mossi du centre (Burkina Faso) ", in Marc Abélès \& Chantal Collard, eds, Âge, pouvoir et société en Afrique noire. Paris, Karthala / Montréal, Presses de l'Université de Montréal : 219-245.

\section{Héritier, Françoise}

1981 L'Exercice de la parenté.

Paris, Gallimard - Le Seuil

("Hautes études»).

1996 Masculin/Féminin. La pensée de la différence. Paris, Odile Jacob.
Houseman, Michael \& Carlo Severi

2009 [1994] Naven ou le donner à voir.

Essai d'interprétation de l'action rituelle.

Paris, CNRs Éd. - Éd. de la MsH

("Chemins de l'ethnologie»).

Houseman, Michael \& Douglas R. White

1996 «Structures réticulaires de la pratique

matrimoniale », L'Homme 139 : 59-86.

Imbs, Françoise

1987 Kumtaabo. Une collectivité rurale

mossi et son rapport à l'espace (Burkina Faso).

Paris, Éd. de l'OrSTOM

("Atlas des structures agraires

au sud du Sahara » 21).

Izard, Michel

1992 L'Odyssée du pouvoir. Un royaume

africain: Etat, société, destin individuel.

Paris, Éd. de l'EHESS ("Recherches

d'histoire et de sciences sociales " 53).

Kohler, Jean-Marie

1971 Activités agricoles

et changements sociaux dans l'Ouest-Mossi.

Paris, ORSTOM, (« Mémoires» 46).

\section{Kroeber, Alfred L.}

1909 "Classificatory Systems of

Relationship ", Journal of the Royal

Anthropological Institute of Great Britain

and Ireland 39: 77-84.

Lallemand, Suzanne

1977 Une famille mossi.

Paris, Éd. du CNRS

(«Recherches voltaïques» 17).

1978 "Le bébé-ancêtre Mossi ",

in Systèmes de signes. Textes réunis

en hommage à Germaine Dieterlen.

Paris, Hermann : 307-316.

1993 La Circulation des enfants

en société traditionnelle. Prêt, don, échange.

Paris, L'Harmattan

( Connaissance des hommes»). 
Laurent, Pierre-Joseph

2009 [2003] Les Pentecôtistes du Burkina

Faso. Mariage, pouvoir et guérison.

Marseille, IRD / Paris, Karthala

(«Hommes et sociétés»).

2010 Beautés imaginaires.

Anthropologie du corps et de la parenté.

Louvain-la-Neuve, Bruylant-Academia

(«Anthropologie prospective» 7).

Lounsbury, Floyd G.

1964 "The Formal Analysis of Crowand Omaha-Type Kinship Terminologies ", in Ward H. Goodenough, ed., Explorations in Cultural Anthropology. Essays in Honor of George Peter Murdock.

New York, McGraw-Hill : 351-394.

Lowie, Robert $\mathrm{H}$.

1928 «A Note on Relationship Terminologies ", American Anthropologist 30 (2) : 263-267.

Needham, Rodney, ed.

1977 La Parenté en question.

Trad. de l'anglais par Martine Karnoouh et Edgar Roskis. Paris, Le Seuil

(«Recherches anthropologiques»).

Nikiéma, Norbert \& Jules Kinda

1997 Dictionnaire orthographique du mooré. Ouagadougou, Sous-commission nationale du mooré.

\section{Poulet, Étienne}

1970 Contribution à l'étude des composantes de la personne humaine chez les Mossi. Poitiers, Université de Poitiers, thèse de doctorat.

Radcliffe-Brown, Alfred R.

1952 Structure and Function in Primitive Society. Essays and Addresses. London, Cohen \& West.

Vinel, Virginie

2000 «Comment les alliés sont aussi des parents : endogamie locale et relations familiales dans un quartier moose sikoomse (Burkina Faso) ", L'Homme 154-155: 205-224.

2005 Des fermmes et des lignages. Ethnologie des relations féminines au Burkina Faso (Moose, Sikoomse). Paris, L'Harmattan («Connaissance des hommes»).

\section{Viveiros de Castro, Eduardo}

2009 Métaphysiques cannibales.

Lignes d'anthropologie post-structurale.

Trad. du portugais (Brésil) par Oiara Bonilla. Paris, Presses universitaires de France (« MétaphysiqueS »).

\section{Zimmermann, Francis}

1993 Enquête sur la parenté.

Paris, Presses universitaires de France

(«Ethnologies»). 
Pierre-Joseph Laurent, Systèmes de mariages et terminologie de parenté chez les Mossi : contribution à l'approche de la terminologie omaha. La terminologie de la parenté mossi, regroupée sous la vaste catégorie omaha, ne porte pas directement sur des indications relatives au mariage, mais sur la manière de mettre en scène des comportements rituels, parfois ambivalents, qui - comme l'indiquent des éléments de contexte - règlent avec minutie les relations des uns avec les autres. Ces relations orientent alors sérieusement les projets d'alliances matrimoniales lesquelles s'insèrent dans un système de mariages qu'il convient d'embrasser d'un seul regard. Le principe de basculement oblique autour d'Ego indique qui vient en remplacement de qui et selon quel statut afin de favoriser le respect des termes des accords passés entre les lignages aux générations précédentes. Par ce processus, ce sont les pactes d'alliance concernant l'octroi d'épouses qui deviennent transmissibles de génération en génération, dès lors que la génération actuelle, qui hérite des accords des aînés, met tout en œuvre pour respecter les comportements adéquats.
Pierre-Joseph Laurent, Marriage Systems and
Kinship Terminology among the Mossi : A Contribution to the Approach Using Omaha Terminology. - Mossi kinship terminology, classified in the vast Omaha category, does not have to do directly with terms for marriage but, instead, with the performance of ritual behaviour that, sometimes ambivalent, scrupulously regulate (as indicated by elements in the context) relations between persons. These relations seriously orient plans for matrimonial alliances as part of a marriage system that should be seen as a whole. The principle of an "oblique switch" around Ego indicates both who is replacing whom and, too, the status involved, the purpose being to enforce the agreements passed between lineages during previous generations. Through this process, the pacts of marital alliance for bestowing women as wives can be transmitted from generation to generation, once the current generation, which has inherited the agreements from its predecessors, does everything necessary to enforce the appropriate behaviour. 